Egyptian

Orthodontic Journal

\title{
ACCURACY AND RELIABILITY OF ORTHODONTIC DENTAL MEASUREMENTS USING DIFFERENT METHODS
}

\author{
Mohamed M. S. Atteya ${ }^{1}$, Abbadi A .El-kadi², \\ Waleed E. Refaat ${ }^{3}$.
}

ABSTRACT:

Objective: to compare the accuracy and reliability of orthodontic dental measurements obtained from CBCT models, digital models and conventional plaster models. Material and methods: The sample consisted of plaster dental models of 30 orthodontic subjects. Dental arch measurements, including mesiodistal widths of teeth and arch widths. Were made with the calipers. The patients were also scanned with CBCT, and measurements were made digitally on a 3-dimensional based dental measurements software (On Demand 3D, Cypermed, Seoul, Korea), Plaster models were scanned with a digital scanner (Canon Pixma MX300, Canon, USA) and measurements were made digitally on Onyx Ceph. Software (Ver. 2.7.18, OnyxCeph, Chemnitz, Germany). Results: For the accuracy evaluation, statistically significant differences were found between conventional models and CBCT models for mesiodistal widths of maxillary right canine, right first molar, left first premolar, left second premolar and left first molar, mandibular right second premolar, left first and premolar, mandibular inter-canine width. Also Statistically significant differences were found between conventional models and digitally scanned models for most mesiodistal teeth widths. However,

1- Canditate, Masters Degree Program, Orthodontic dept., Faculty of Dentistry, Suez Canal University.

2- Professor, Orthodontic dept. Faculty of Dentistry, Suez Canal University.

3- Assisstant Professor, Orthodontic dept., Faculty of Dentistry, Suez Canal University. 
Egyptian

Orthodontic Journal

the mean differences between the measurements were ranging from $-0.222-0.211 \mathrm{~mm}$ which is not clinically significant. For reliability evaluation results showed excellent agreement for the three methods between measurements made at three different time intervals. Conclusion: Digital CBCT models and digitally scanned plaster models used in this study are as accurate as conventional models and can be a good alternative. The three methods used in this study are highly reliable.

Key Words: CBCT; Mesio-distal teeth width; Arch width.

\section{INTRODUCTION}

Successful orthodontic treatment is based on extensive diagnosis and treatment planning. Dental models, photographs, radiographs and clinical examinations provide essential information for diagnosis. ${ }^{1}$ Orthodontic study models are an important part of diagnosis, case presentation, treatment planning, evaluation of treatment progress and record keeping. ${ }^{1-2}$ Digital photographs and radiographs are now routinely incorporated into electronic files but digital dental models are not as widely used. Electronic storage of patient information including study models eliminates problems of physical storage, retrieval, maintenance, and office management. Documentation of treatment progress and communication between professional colleagues can be enhanced by digital records. ${ }^{3}$ Several attempts have been made to transform dental plaster models into 3-dimensional (3D) virtual models. ${ }^{4}$

Several studies were conducted to compare 3D virtual models with plaster models. Santoro et $\mathrm{al}^{5}$ compared measurements obtained from OrthoCAD to those made on traditional models. OrthoCAD values were found to be a clinically acceptable alternative to plaster casts.

Whetten et $\mathrm{al}^{6}$ investigated the difference between plaster models and the virtual 3D model (e-model) in treatment planning for Class II patients. They concluded that digital orthodontic study models (e-models) were a valid alternative to traditional plaster models in treatment planning for these patients. 
Egyptian

Orthodontic Journal

Zanaty et $\mathrm{al}^{7}$ compared the accuracy of dental measurements on plaster dental casts with those from computed tomography scans of the dentition. Dental arch measurements including mesio-distal widths of teeth, arch widths, arch lengths, arch perimeters, and palatal depths. Results showed excellent agreement in most measurements between the 2 methods in the 3 planes of space.

The present work will then compare CBCT dental measurements and measurements on digitally scanned plaster models to conventional plaster models (as a gold standard).

\section{MATERIALS AND METHODS}

Our sample consisted of 30 adults, their ages ranged from 16 to 28 years. The subjects were outpatients selected from the orthodontic clinic at the Faculty of dentistry, Suez Canal University. All subjects had erupted permanent dentitions from the first molar of one side to the first molar of the other side with no orthodontic appliances. Measurements were recorded on the conventional model, the $3 \mathrm{D}$ virtual model from the CBCT scan and digitally scanned plaster models with a blind protocol.

For the conventional plaster models, the maxillary and mandibular casts of the 30 subjects were measured with Vernier calipers (Mitutoyo, Digimatic, Kawasaki, Japan.) calibrated to the nearest $0.1 \mathrm{~mm}$.

For the 3D virtual models, each subject's head was scanned with a CBCT device Cranex 3D (Soredex, Tuusula, Finland). At an axial section of $0.3 \mathrm{~mm}$. The reconstruction interval was done with $1 \mathrm{~mm}$. and total rotation time was 24 seconds. The anode current was $10 \mathrm{~mA}$, and anode voltage $57-90 \mathrm{kV}$. During scanning, the patient should bite on three tongue depressor sticks to keep the maxillary and mandibular teeth separated. Separation was necessary to reproduce occlusal anatomy and avoid blurring of the dental images. The inclination of the gantry was set parallel to the maxillary occlusal plane in all cases. All data were saved with a DICOM extension.

The software OnDemand3D software (Cypermed, Seoul, Korea) was used for image reconstruction from the Axial CT-scans. 
Egyptian

Orthodontic Journal

The 3D CBCT models were manipulated using analysis tools provided by OnDemand3D software. The resulting digital cast can be viewed on the computer at any angle or position. This allows direct measurements of distances, volumes, and angles.

The tool 3D ruler was used to measure distances on 3D volumes. After marking two points, the computer calculated the shortest distance between them to the nearest $0.1 \mathrm{~mm}$.

For the plaster casts were scanned with Canon Pixma MX300 (Canon, USA) scanner after marking the landmarks to be measured using a black permanent marker. A millimeter ruler was scanned with each cast to scale the casts for accurate measurements. The ruler must be perfectly aligned horizontally with the $\mathrm{X}$-axis to ensure accurate scaling.

Images were manipulated using Onyx Ceph. Software (ver. 2.7.18, OnyxCeph, Chemnitz, Germany).

The following dental arch measurements were taken for both the conventional casts and the $3 \mathrm{D}$ virtual models by the same observer (M.M.S.A.):

1. Mesiodistal tooth width from the first permanent molar of 1 side to the first permanent molar of the other side (Fig. 1,2\&3).

2. Arch width, maxillary inter canine width, mandibular inter canine width, maxillary inter first molar width and mandibular inter first molar width ${ }^{8}$ (Fig.4-5).

Replication of the measurements (for reliability testing) was done by the same observer (M.M.S.A.). These intraobserver measurements were made at a time interval of one week and two weeks for the three methods, a week after the first set of measurements.

Accuracy was considered as the extent to which measurements made on CBCT and the digitally scanned models are compared to the conventional plaster models. While reliability was estimated as the extent to which measurements were repeatable under same conditions for the three methods. In this study one examiner (M.M.S.A) carried out all the measurements and so only intra-examiner variation was assessed. 


\section{Egyptian}

Orthodontic Journal

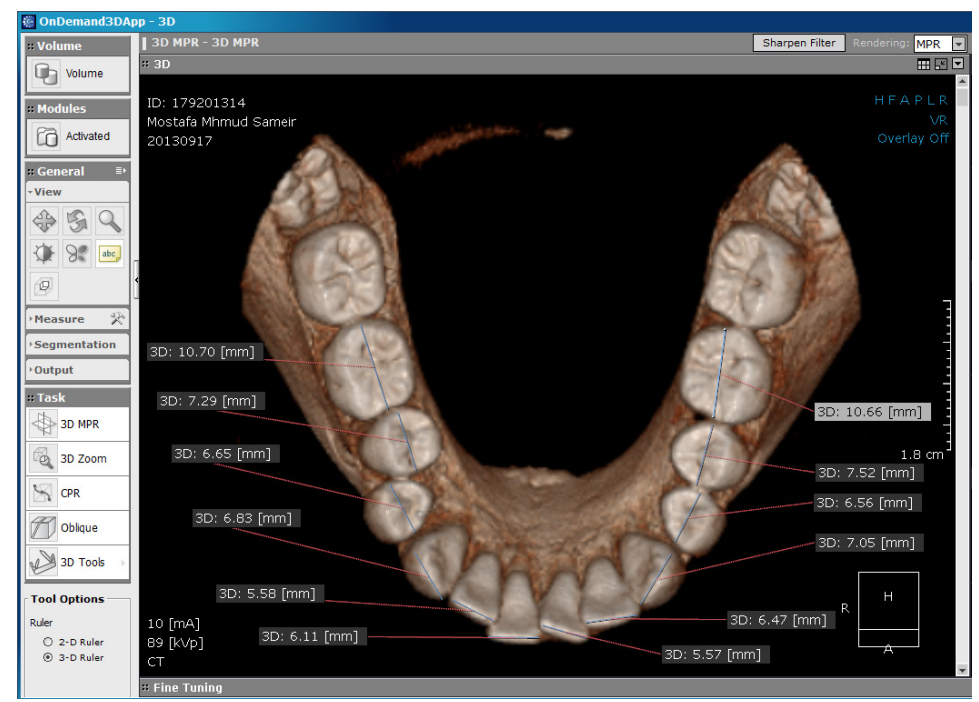

Figure 1: Illustration showing maxillary mesio-distal teeth width measurement on CBCT models.

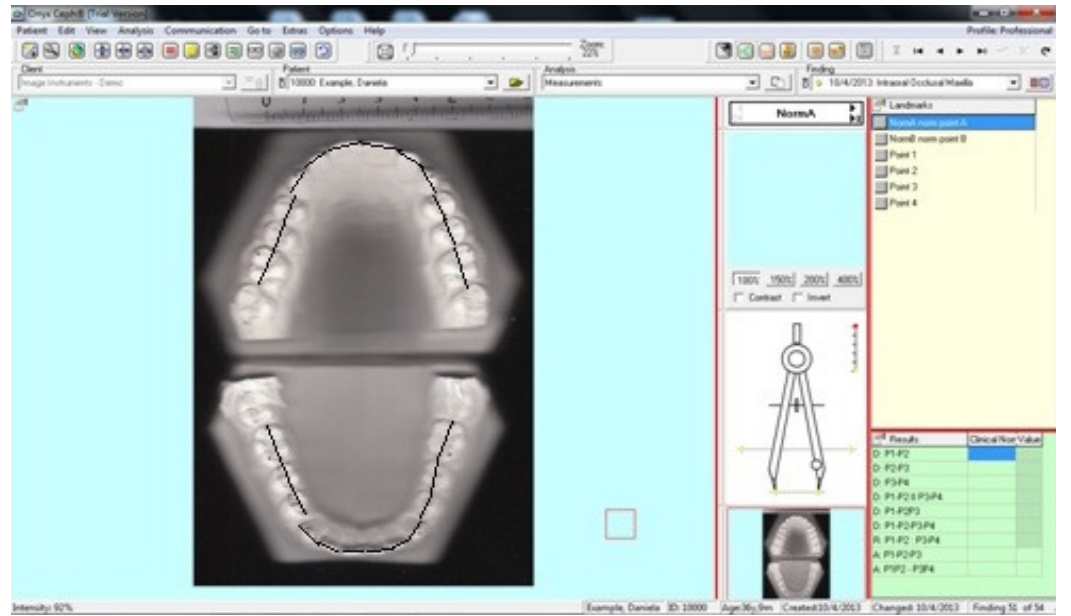

Figure2: Illustration showing mandibular mesio-distal teeth width measurement on CBCT models.

Volume 45 - June 2014 


\section{Egyptian}

Orthodontic Journal

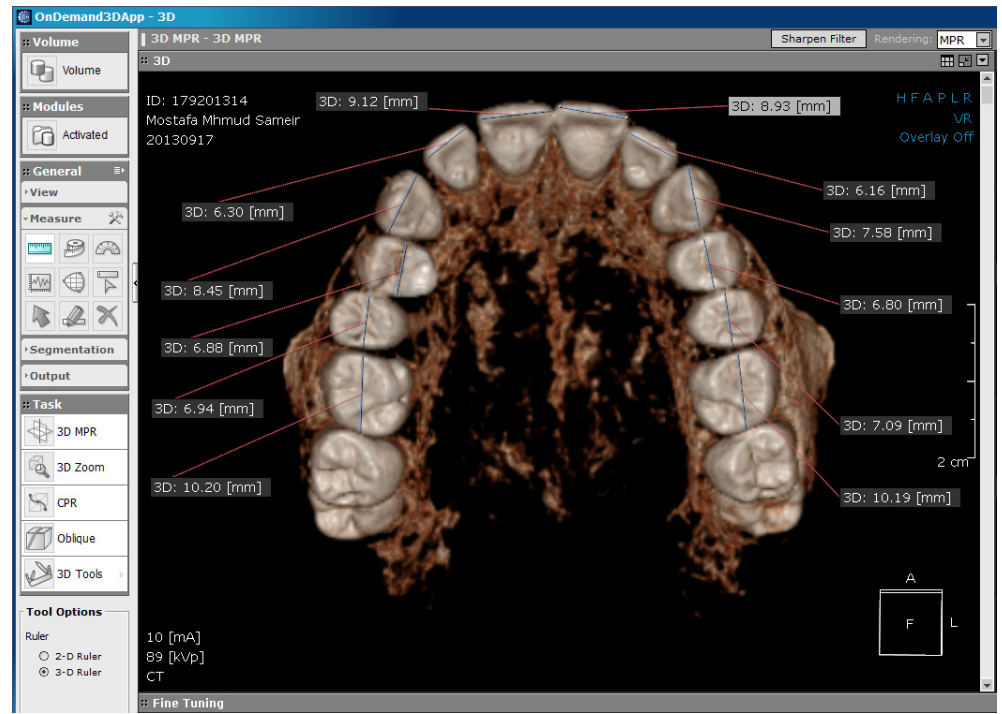

Figure3: Illustration showing mesio-distal teeth width measurement on digitally scanned plaster models.

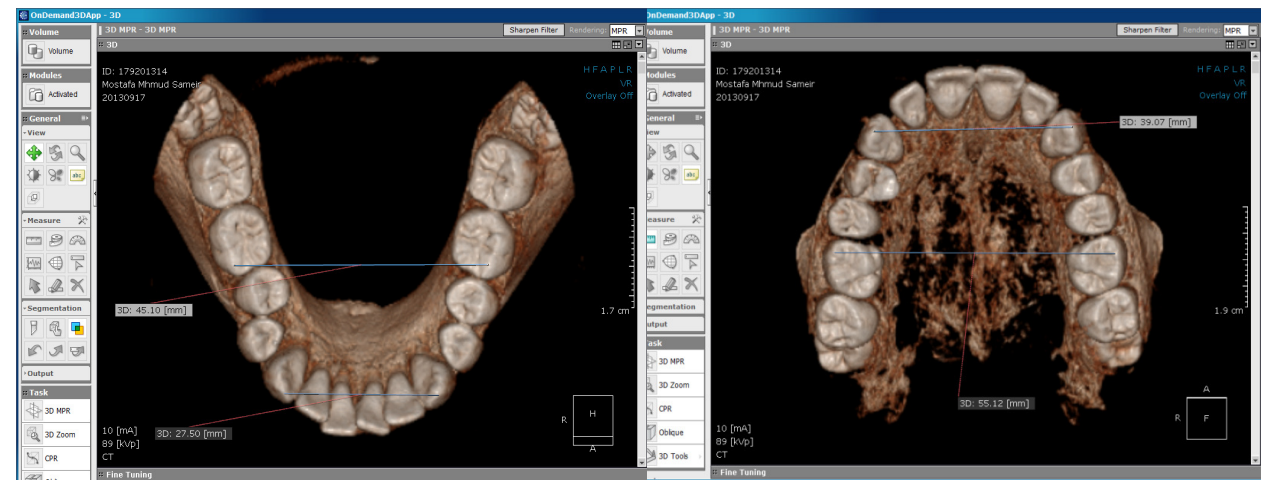

Figure 4: Illustration showing inter-canine and inter-molar width measurements on CBCT models. 


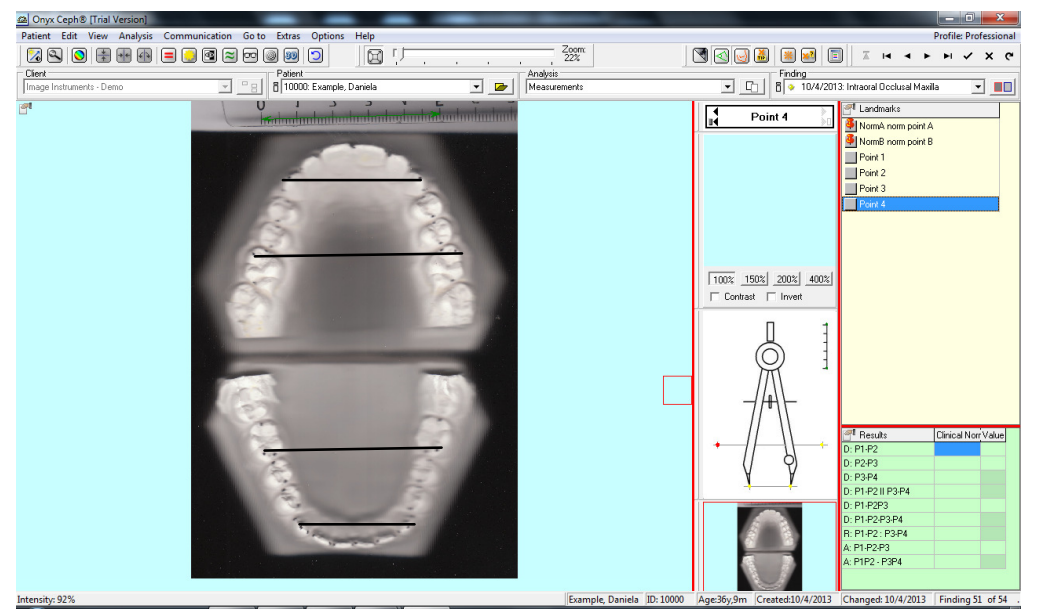

Figure 5: Illustration showing inter-canine and inter-molar widths measurement on digitally scanned plaster models.

\section{STATISTICAL ANALYSIS}

The data were collected, tabulated, and analyzed. Paired t test was used to test the Accuracy between different measuring methods. Intraclass correlation coefficient was used to test the reliability within each method. ${ }^{9}$ ICC values were interpreted as follows: $>0.75$ was excellent, $0.40-0.75$ was fair to good and $<0.40$ was poor. ${ }^{10}$

\section{RESULTS}

For the CBCT models measurements of the mesiodistal widths of most maxillary and mandibular teeth showed statistically insignificant differences between conventional models and CBCT models (Table 1-2) (Fig. 6,7,8\&9). The mesiodistal measurements of the following teeth showed statistically significant differences between the 2 measuring modalities: maxillary right canine $(\mathrm{p} \leq 0.01)$, maxillary right first molar $(\mathrm{p} \leq 0.05)$, maxillary left first premolar $(\mathrm{p} \leq 0.01)$, maxillary left second premolar $(p \leq 0.05)$, maxillary left first molar $(p \leq 0.001)$, mandibular right second premolar $(\mathrm{p} \leq 0.05)$ and left first premolar $(\mathrm{p} \leq 0.001)$. Mandibular inter-canine width showed statistically significant difference $(p \leq 0.001)$ between the 2 measuring modalities (Table 3) (Fig. 10). 


\section{Egyptian}

Orthodontic Journal

Table (1): Maxillary mesio-distal teeth width measured on conventional models compared to maxillary mesio-distal teeth width measured on CBCT models.

\begin{tabular}{|c|c|c|c|c|c|c|c|}
\hline \multirow[b]{2}{*}{ Tooth } & \multicolumn{2}{|c|}{$\begin{array}{c}\text { Conventional } \\
\text { models }\end{array}$} & \multicolumn{2}{|c|}{ CBCT models } & & & \\
\hline & Mean & S.D. & Mean & S.D. & $\begin{array}{c}\text { Mean } \\
\text { Diff. }\end{array}$ & $\begin{array}{l}\text { S.E. } \\
\text { Diff. }\end{array}$ & $\mathrm{P}$ \\
\hline UR1 & 8.689 & 0.664 & 8.633 & 0.800 & 0.056 & 0.056 & $0.320 \mathrm{NS}$ \\
\hline UR2 & 6.578 & 0.734 & 6.544 & 0.706 & 0.033 & 0.046 & $0.470 \mathrm{NS}$ \\
\hline UR3 & 7.678 & 0.615 & 7.522 & 0.640 & 0.156 & 0.057 & $0.007 * *$ \\
\hline UR4 & 6.467 & 0.545 & 6.422 & 0.653 & 0.044 & 0.055 & $0.417 \mathrm{NS}$ \\
\hline UR5 & 6.422 & 0.519 & 6.389 & 0.594 & 0.033 & 0.040 & $0.408 \mathrm{NS}$ \\
\hline UR6 & 9.978 & 0.599 & 9.867 & 0.545 & 0.111 & 0.046 & $0.018 *$ \\
\hline UL1 & 8.678 & 0.762 & 8.711 & 0.753 & -0.033 & 0.037 & $0.369 \mathrm{NS}$ \\
\hline UL2 & 6.556 & 0.736 & 6.511 & 0.691 & 0.044 & 0.047 & $0.349 \mathrm{NS}$ \\
\hline UL3 & 7.622 & 0.532 & 7.567 & 0.498 & 0.056 & 0.048 & $0.254 \mathrm{NS}$ \\
\hline UL4 & 6.556 & 0.500 & 6.400 & 0.557 & 0.156 & 0.050 & $0.002 * *$ \\
\hline UL5 & 6.611 & 0.698 & 6.511 & 0.658 & 0.100 & 0.045 & $0.028 *$ \\
\hline UL6 & 10.111 & 0.678 & 9.900 & 0.562 & 0.211 & 0.056 & $0.001 * * *$ \\
\hline
\end{tabular}

S.D. $=$ Standard deviation

S.E. $=$ Standard error.

Diff. $=$ Difference.

$\mathrm{P}=$ Probability level for the paired comparison between conventional models and CBCT (Paired $\mathrm{t}$ test).

NS $=$ Insignificant $(\mathrm{p}>0.05)$.

* = Significant at $\mathrm{p} \leq 0.05$

$* * *=$ Significant at $\mathrm{p} \leq 0.001$

$* *=$ Significant at $\mathrm{p} \leq 0.01$

$\mathrm{UR}=$ Upper right.

$\mathrm{UL}=$ Upper Left.

Volume 45 - June 2014 


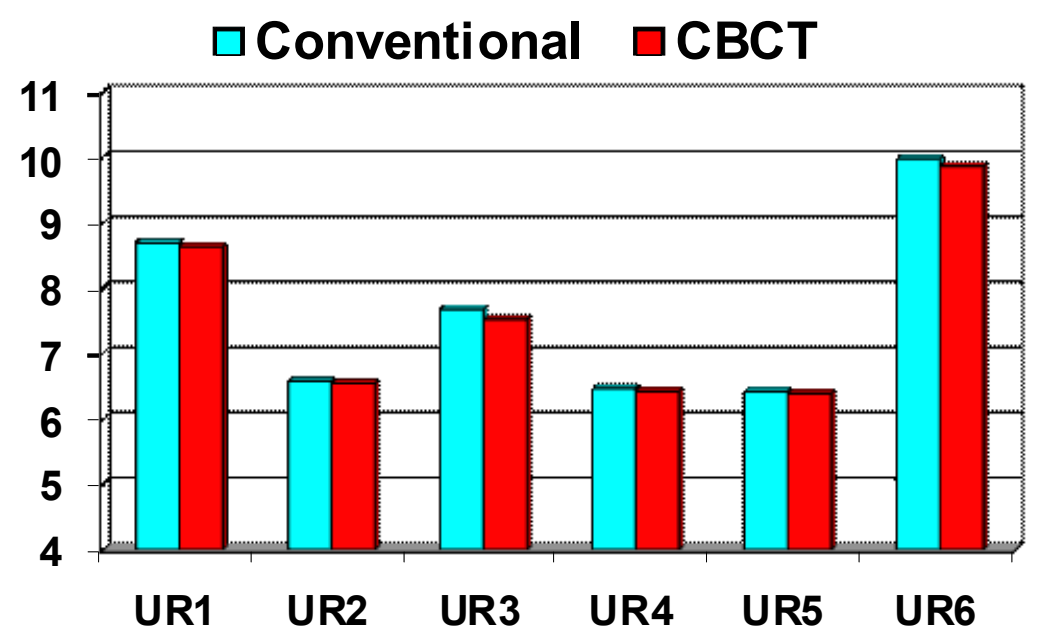

Figure 6: Histogram showing mean upper right mesio-distal tooth width measured on conventional models and CBCT models.

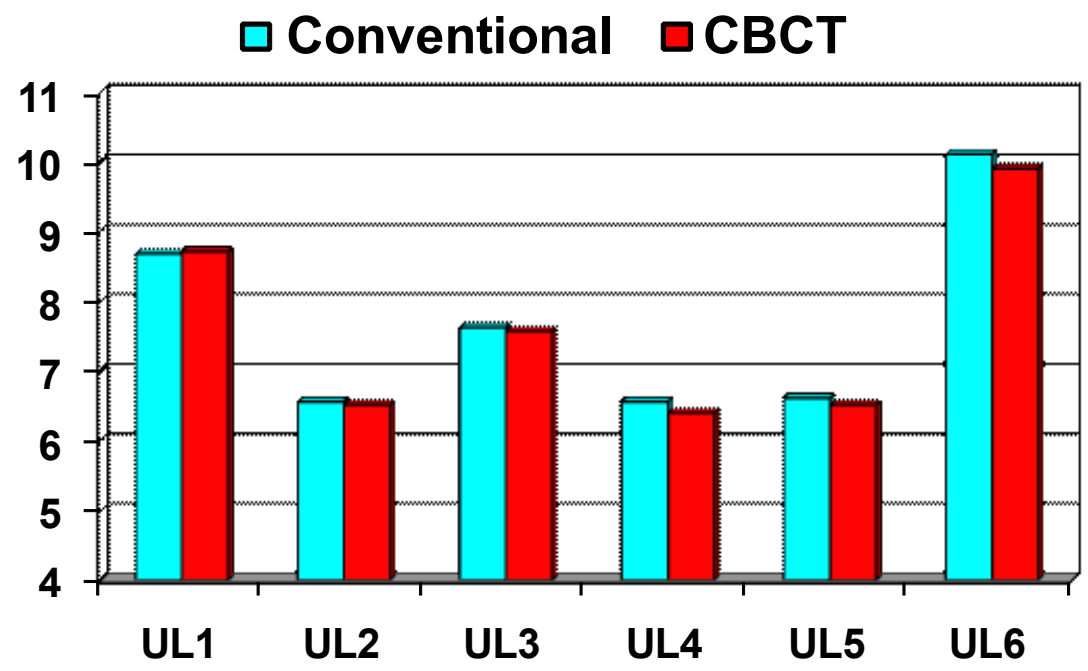

Figure 7: Histogram showing mean upper left mesio-distal tooth width measured on conventional models and CBCT models. 


\section{Egyptian}

Orthodontic Journal

Table (2): Mandibular mesio-distal teeth width measured on conventional models compared to mandibular mesio-distal teeth width measured on CBCT models.

\begin{tabular}{|c|c|c|c|c|c|c|c|}
\hline \multirow[b]{2}{*}{ Tooth } & \multicolumn{2}{|c|}{$\begin{array}{c}\text { Conventional } \\
\text { models }\end{array}$} & \multicolumn{2}{|c|}{$\mathrm{CBCT}$} & \multirow[b]{2}{*}{$\begin{array}{l}\text { Mean } \\
\text { Diff. }\end{array}$} & \multirow[b]{2}{*}{$\begin{array}{l}\text { S.E. } \\
\text { Diff. }\end{array}$} & \multirow[b]{2}{*}{$\mathrm{P}$} \\
\hline & Mean & S.D. & Mean & S.D. & & & \\
\hline LR1 & 5.400 & 0.493 & 5.422 & 0.540 & -0.022 & 0.047 & $0.640 \mathrm{NS}$ \\
\hline LR2 & 5.889 & 0.550 & 5.800 & 0.565 & 0.089 & 0.046 & $0.059 \mathrm{NS}$ \\
\hline LR3 & 6.700 & 0.661 & 6.644 & 0.624 & 0.056 & 0.040 & $0.167 \mathrm{NS}$ \\
\hline LR4 & 6.978 & 0.749 & 6.900 & 0.619 & 0.078 & 0.067 & $0.252 \mathrm{NS}$ \\
\hline LR5 & 7.189 & 0.748 & 7.089 & 0.788 & 0.100 & 0.050 & $0.049 *$ \\
\hline LR6 & 10.256 & 0.815 & 10.211 & 0.786 & 0.044 & 0.035 & $0.208 \mathrm{NS}$ \\
\hline LL1 & 5.400 & 0.493 & 5.433 & 0.562 & -0.033 & 0.037 & $0.369 \mathrm{NS}$ \\
\hline LL2 & 5.900 & 0.582 & 5.889 & 0.589 & 0.011 & 0.043 & $0.798 \mathrm{NS}$ \\
\hline LL3 & 6.667 & 0.636 & 6.700 & 0.710 & -0.033 & 0.049 & $0.494 \mathrm{NS}$ \\
\hline LL4 & 7.011 & 0.679 & 6.811 & 0.634 & 0.200 & 0.048 & $0.001 * * *$ \\
\hline LL5 & 7.056 & 0.784 & 7.011 & 0.757 & 0.044 & 0.047 & $0.349 \mathrm{NS}$ \\
\hline LL6 & 10.278 & 0.600 & 10.278 & 0.475 & 0.000 & 0.045 & $1.000 \mathrm{NS}$ \\
\hline
\end{tabular}

S.D. $=$ Standard deviation.

S.E. $=$ Standard error.

Diff. $=$ Difference

$\mathrm{P} \quad=$ Probability level for the paired comparison between conventional models and CBCT (Paired t test).

NS $=$ Insignificant $(\mathrm{p}>0.05)$.

* $\quad=$ Significant at $\mathrm{p} \leq 0.05$

$* *=$ Significant at $\mathrm{p} \leq 0.01$

$* * *=$ Significant at $\mathrm{p} \leq 0.001$

$\mathrm{LR}=$ Lower right.

$\mathrm{LL}=$ Lower Left.

Volume 45 - June 2014 


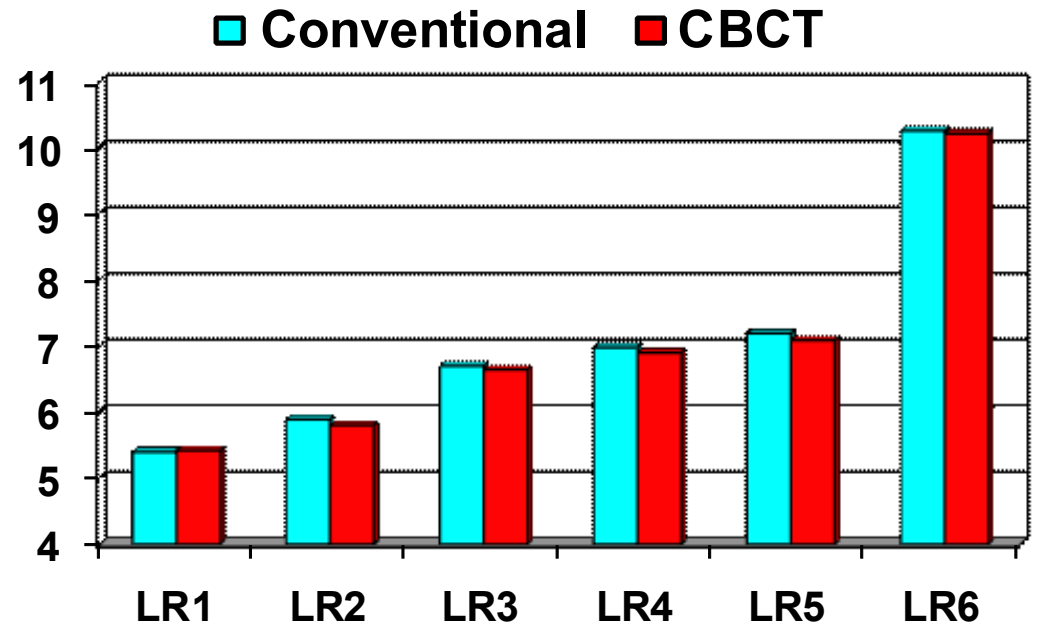

Figure 8: Histogram showing mean lower right mesio-distal tooth width measured on conventional models and CBCT

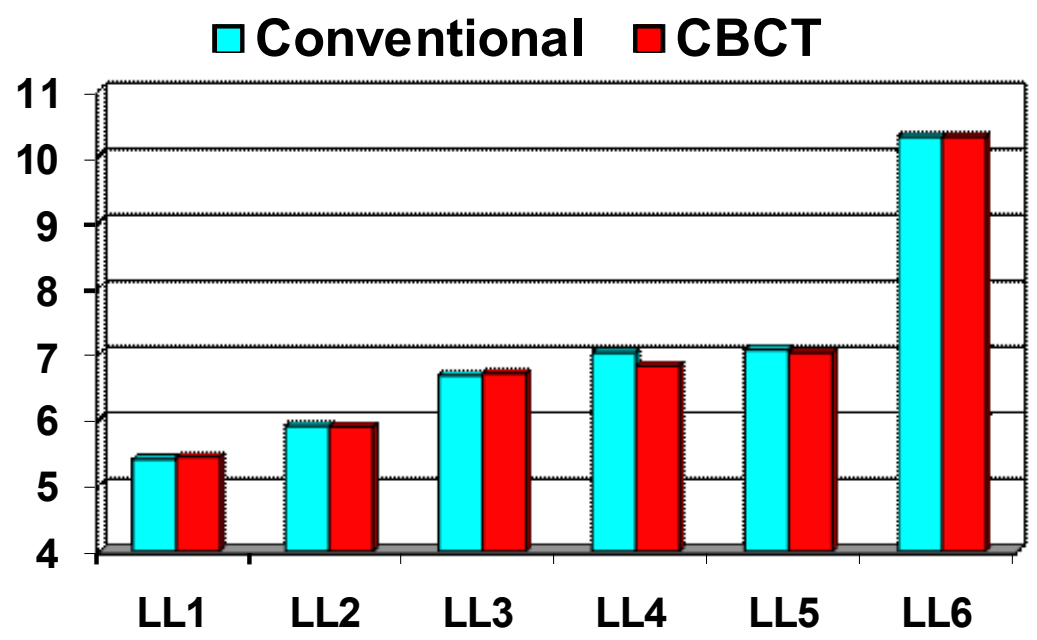

Figure 9: Histogram showing mean lower left mesio-distal tooth width measured on conventional models and CBCT models. 
Table (3):Mandibular Arch Widths on Conventional Models Compared to Mandibular Arch Widths on CBCT Models.

\begin{tabular}{|l|c|c|c|c|c|c|c|}
\cline { 2 - 8 } \multicolumn{1}{c|}{} & \multicolumn{2}{c|}{$\begin{array}{c}\text { Conventional } \\
\text { models }\end{array}$} & \multicolumn{2}{c|}{ CBCT } & \multicolumn{2}{c|}{} \\
\hline Arch width & Mean & S.D. & Mean & S.D. & $\begin{array}{c}\text { Mean } \\
\text { Diff. }\end{array}$ & $\begin{array}{c}\text { S.E. } \\
\text { Diff. }\end{array}$ & P \\
\hline ICW & 25.922 & 2.328 & 26.133 & 2.371 & -0.211 & 0.064 & $0.001 * * *$ \\
\hline IMW & 43.456 & 3.474 & 43.311 & 3.618 & 0.144 & 0.104 & $0.170 \mathrm{NS}$ \\
\hline
\end{tabular}

S.D .= Standard deviation.

S.E. $=$ Standard error.

Diff. $=$ Difference.

$\mathrm{P}=$ Probability level for the paired comparison between conventional models and CBCT (Paired $\mathrm{t}$ test).

NS = Insignificant $(\mathrm{p}>0.05)$.

* $\quad=$ Significant at $\mathrm{p} \leq 0.05$

** $=$ Significant at $\mathrm{p} \leq 0.01$

$* * *=$ Significant at $\mathrm{p} \leq 0.001$

$\mathrm{ICW}=$ Inter-canine Width

IMW= Inter-molar Width.

$\square$ Conventional

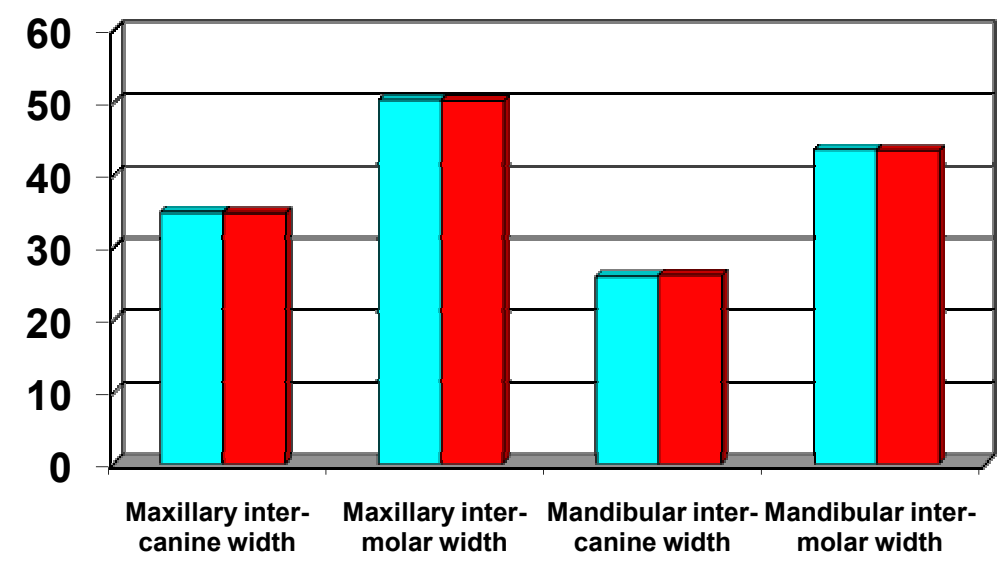

Figure 10: Histogram showing mean lower left mesio-distal tooth width measured on conventional models and CBCT models. 
Egyptian

Orthodontic Journal

For the digitally scanned plaster models measurements of the mesiodistal widths of most maxillary and mandibular teeth showed statistically significant differences between conventional models and digitally scanned plaster models (Table 5-6) (Fig. 11,12,13\&14). The mesiodistal measurements of the following teeth showed statistically insignificant differences between the 2 measuring modalities: maxillary left first premolar. maxillary left second premolar, mandibular right lateral incisor, mandibular right second premolar, mandibular left lateral incisor, mandibular left canine and mandibular left second premolar.

Intra-observer error assessment excellent correlation between the intra-observer measurements for the conventional, CBCT and digitally scanned models measuring techniques. The intraobserver values for the conventional models ranged from 0.815 to 0.997 . The values for the CBCT models ranged from 0.829 to 0.999 . The values for the digitally scanned models ranged from 0.984 to 1.000 .

\section{DISCUSSION}

Dental study models are an important armamentarium used by orthodontists for diagnosis and treatment planning. The purpose of this study was to compare the accuracy and reliability of dental measurements obtained from CBCT models and digitally scanned models with those that were taken manually using digital Vernier caliper on conventional plaster models.

\section{CBCT models:}

Linear measurements of maxillary and mandibular arches revealed statistically insignificant differences between the conventional and CBCT models for most measuremets, while revealed statistically significant differences for some measurements. The practical significance of this discrepancy is questionable. According to Proffit, Fields and Sarver ${ }^{11}$ 2007 a tooth-size discrepancy less than $1.5 \mathrm{~mm}$ is rarely considered significant. The mean differences between both methods were ranging from $-0.211-0.211 \mathrm{~mm}$ which is not clinically significant. 
Egyptian

Orthodontic Journal

The CBCT values tended to slightly underestimate the real value for most measurements. This seemed to make less significant differences for single measurements which become more significant for compound measurements. This finding is in agreement with Baumgaeretel ${ }^{8} 2009$, Brown $^{12}$ et al and Lascala ${ }^{13}$ et al who also found slight underestimation of CBCT values for most measurements.

One of the greatest sources of random error was no physical barrier to the placement of measurement points with virtual models (Naidu and Freer ${ }^{14}$ 2013) due to viewing a 3D structure as a two dimensional image where convex structures of teeth, curve of spee, inclination and rotation may influence measurements $\left(Z_{\text {Zilberman }}{ }^{15}\right.$ et al, Tehranchi ${ }^{16}$ et al). Also reduction in image quality due to soft-tissue attenuation, metallic artifacts and patient motion may be a cause (Periago ${ }^{17}$ et al). Another cause may be the difference between the natures of the two methods. The CBCT models are measured on a computer screen using digital tools on enlarged image which differs from the traditional Vernier caliper.

\section{Digitally scanned plaster models:}

Linear measurements of maxillary and mandibular arches revealed statistically significant differences between the conventional and digitally scanned models for most measuremets, while revealed statistically insignificant differences for some measurements. The mean differences between both methods were ranging from $-0.222-0.056 \mathrm{~mm}$ which is not clinically significant.

The digitally scanned models values tended to slightly overestimate the real value for most measurements. This finding agrees with Champagne ${ }^{18}$ who found magnification in some measurements. On the other hand these findings disagrees with Mayers ${ }^{19}$ et al who showed no significant differences between plaster and digital models.

This over estimation may be due to magnifying cast image through the scanner or the difference between the natures of the two methods. The digitally scanned models are measured on a computer screen using digital tools on enlarged image which differs from the traditional Vernier caliper. 


\section{Egyptian}

Orthodontic Journal

Table (5): Maxillary mesio-distal teeth width measured on conventional models compared to maxillary mesio-distal teeth width measured on digitally scanned models.

\begin{tabular}{|c|c|c|c|c|c|c|c|}
\hline \multirow[b]{2}{*}{ Tooth } & \multicolumn{2}{|c|}{$\begin{array}{l}\text { Conventional } \\
\text { models }\end{array}$} & \multicolumn{2}{|c|}{ Scanner } & & & \\
\hline & Mean & S.D. & Mean & S.D. & $\begin{array}{l}\text { Mean } \\
\text { Diff. }\end{array}$ & $\begin{array}{l}\text { S.E. } \\
\text { Diff. }\end{array}$ & $\mathrm{P}$ \\
\hline UR1 & 8.689 & 0.664 & 8.833 & 0.691 & -0.144 & 0.046 & $0.002 * *$ \\
\hline UR2 & 6.578 & 0.734 & 6.800 & 0.796 & -0.222 & 0.044 & $0.001 * * *$ \\
\hline UR3 & 7.678 & 0.615 & 7.867 & 0.565 & -0.189 & 0.041 & $0.001 * * *$ \\
\hline UR4 & 6.467 & 0.545 & 6.622 & 0.552 & -0.156 & 0.052 & $0.004 * *$ \\
\hline UR5 & 6.422 & 0.519 & 6.533 & 0.622 & -0.111 & 0.049 & $0.025 *$ \\
\hline UR6 & 9.978 & 0.599 & 10.122 & 0.493 & -0.144 & 0.043 & $0.001 * * *$ \\
\hline UL1 & 8.678 & 0.762 & 8.822 & 0.815 & -0.144 & 0.040 & $0.001 * * *$ \\
\hline UL2 & 6.556 & 0.736 & 6.733 & 0.776 & -0.178 & 0.049 & $0.001 * * *$ \\
\hline UL3 & 7.622 & 0.532 & 7.756 & 0.504 & -0.133 & 0.048 & $0.007 * *$ \\
\hline UL4 & 6.556 & 0.500 & 6.567 & 0.498 & -0.011 & 0.037 & $0.765 \mathrm{NS}$ \\
\hline UL5 & 6.611 & 0.698 & 6.589 & 0.669 & 0.022 & 0.032 & $0.483 \mathrm{NS}$ \\
\hline UL6 & 10.111 & 0.678 & 10.300 & 0.589 & -0.189 & 0.052 & $0.001 * * *$ \\
\hline
\end{tabular}

S.D. $=$ Standard deviation

S.E. $=$ Standard error.

Diff. $=$ Difference.

$\mathrm{P}=$ Probability level for the paired comparison between conventional models and CBCT (Paired $\mathrm{t}$ test).

$\mathrm{NS}=$ Insignificant $(\mathrm{p}>0.05)$.

$*=$ Significant at $\mathrm{p} \leq 0.05$

$* *=$ Significant at $\mathrm{p} \leq 0.01$

$* * *=$ Significant at $\mathrm{p} \leq 0.001$

Volume 45 - June 2014 


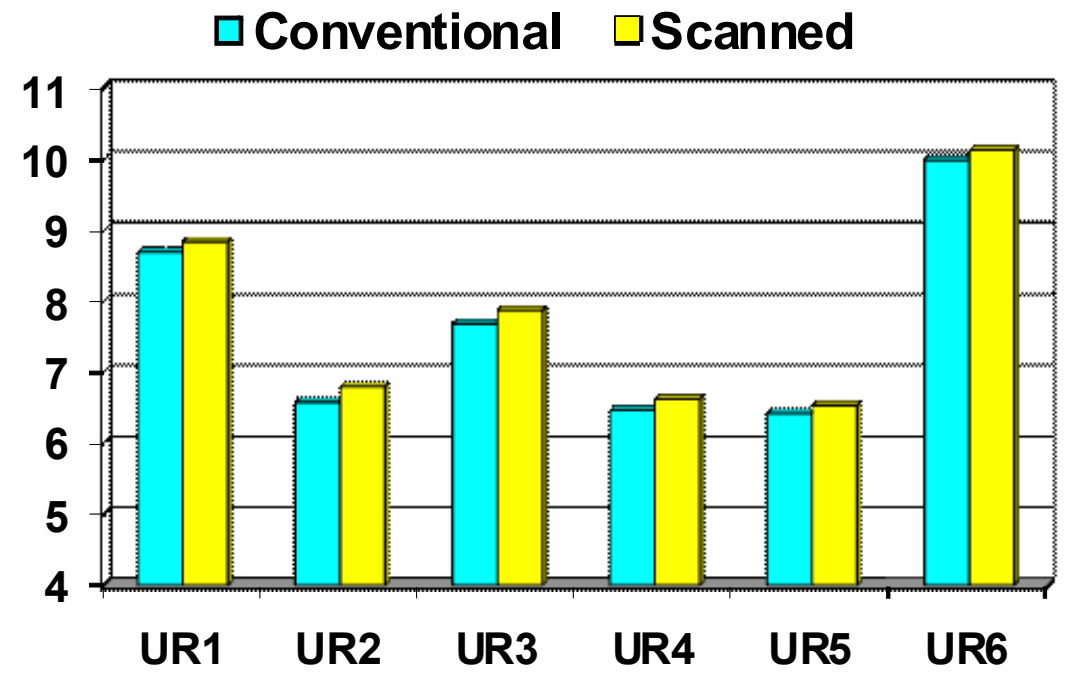

Figure 11: Histogram showing mean upper right mesio-distal tooth width measured on conventional models and digitally scanned models.

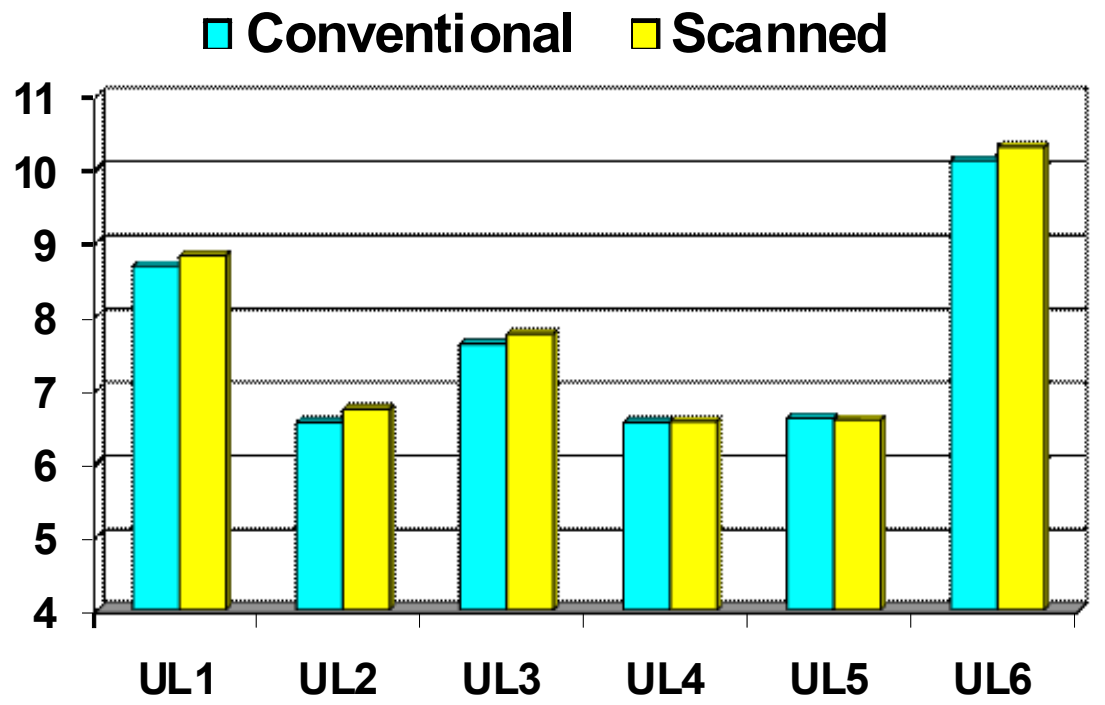

Figure 12: Histogram showing mean upper left mesio-distal tooth width measured on conventional models and digitally scanned models. 


\section{Egyptian}

Orthodontic Journal

Table (6): Mandibular mesio-distal teeth width measured on conventional models compared to mandibular mesio-distal teeth width measured on digitally scanned models.

\begin{tabular}{|c|c|c|c|c|c|c|c|}
\hline \multirow[b]{2}{*}{ Tooth } & \multicolumn{2}{|c|}{$\begin{array}{l}\text { Conventional } \\
\text { models }\end{array}$} & \multicolumn{2}{|c|}{ Scanner } & & & \\
\hline & Mean & S.D. & Mean & S.D. & $\begin{array}{l}\text { Mean } \\
\text { Diff. }\end{array}$ & $\begin{array}{l}\text { S.E. } \\
\text { Diff. }\end{array}$ & $\mathrm{P}$ \\
\hline LR1 & 5.400 & 0.493 & 5.489 & 0.503 & -0.089 & 0.044 & $0.045 *$ \\
\hline LR2 & 5.889 & 0.550 & 5.822 & 0.510 & 0.067 & 0.041 & $0.109 \mathrm{NS}$ \\
\hline LR3 & 6.700 & 0.661 & 6.633 & 0.608 & 0.067 & 0.026 & $0.013 *$ \\
\hline LR4 & 6.978 & 0.749 & 7.122 & 0.805 & -0.144 & 0.049 & $0.004 * *$ \\
\hline LR5 & 7.189 & 0.748 & 7.167 & 0.691 & 0.022 & 0.042 & $0.596 \mathrm{NS}$ \\
\hline LR6 & 10.256 & 0.815 & 10.433 & 0.765 & -0.178 & 0.049 & $0.001 * * *$ \\
\hline LL1 & 5.400 & 0.493 & 5.522 & 0.565 & -0.122 & 0.041 & $0.004 * *$ \\
\hline LL2 & 5.900 & 0.582 & 5.900 & 0.542 & 0.000 & 0.045 & $1.000 \mathrm{NS}$ \\
\hline LL3 & 6.667 & 0.636 & 6.622 & 0.610 & 0.044 & 0.038 & $0.250 \mathrm{NS}$ \\
\hline LL4 & 7.011 & 0.679 & 7.189 & 0.652 & -0.178 & 0.046 & $0.001 * * *$ \\
\hline LL5 & 7.056 & 0.784 & 7.067 & 0.731 & -0.011 & 0.043 & $0.798 \mathrm{NS}$ \\
\hline LL6 & 10.278 & 0.600 & 10.389 & 0.575 & -0.111 & 0.040 & $0.007 * *$ \\
\hline
\end{tabular}

S.D. $=$ Standard deviation.

S.E. $=$ Standard error.

Diff. $=$ Difference

$\mathrm{P} \quad=$ Probability level for the paired comparison between conventional models and CBCT (Paired $t$ test).

$\mathrm{NS}=$ Insignificant $(\mathrm{p}>0.05)$

* $\quad=$ Significant at $\mathrm{p} \leq 0.05$

$* *=$ Significant at $\mathrm{p} \leq 0.01$

$* * *=$ Significant at $\mathrm{p} \leq 0.001$

Volume 45 - June 2014 
$\square$ Conventional $\square$ Scanned

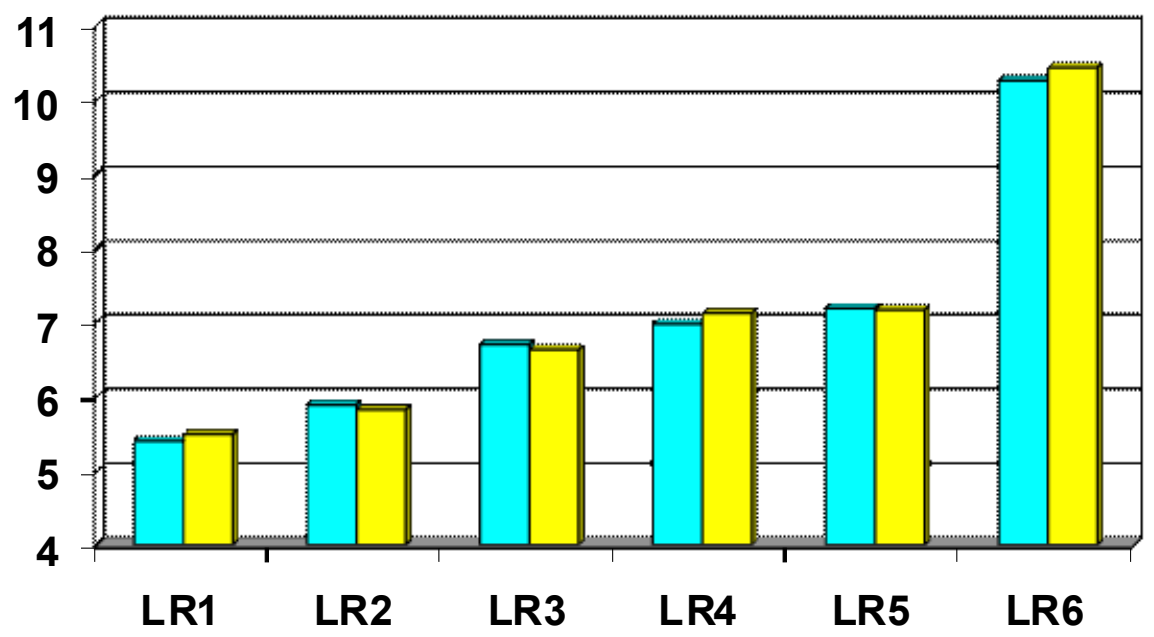

Figure 13: Histogram showing mean lower right mesio-distal tooth width measured on conventional models and digitally scanned models.

$\square$ Conventional $\square$ Scanned

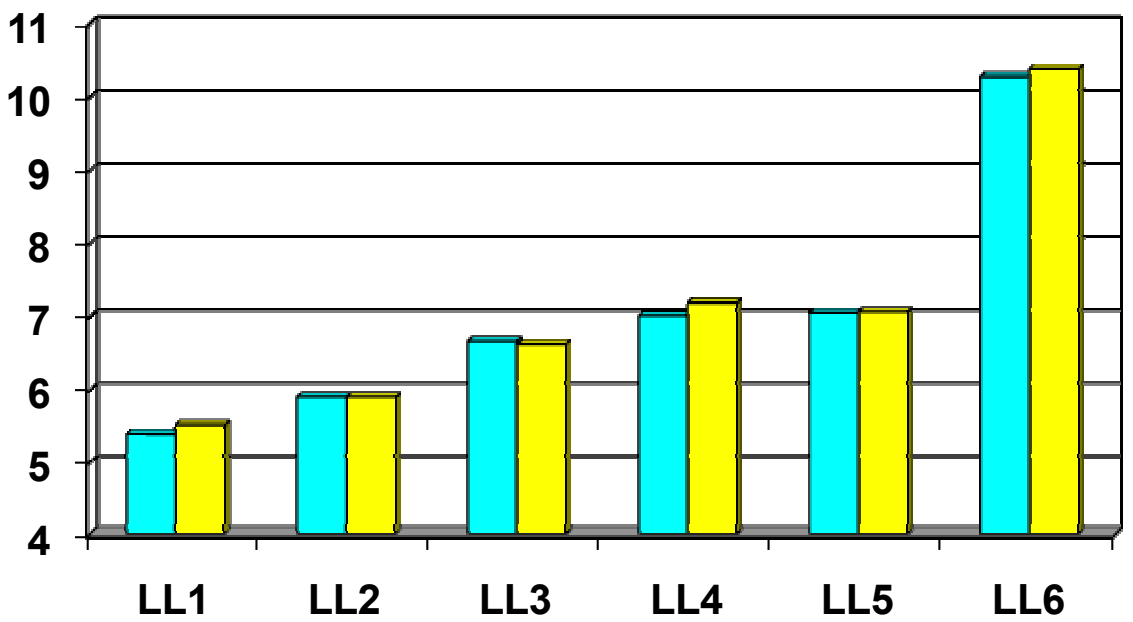

Figure 14: Histogram showing mean lower left mesio-distal tooth width measured on conventional models and digitally scanned models. 
Egyptian

Orthodontic Journal

\section{REFERENCES}

1. Quimby ML, Vig KW, Rashid RG, Firestone AR. The accuracy and reliability of measurements made on computer-based digital models. Angle Orthod. 2004;74:298-303.

2. Ryden H., Bjelkhagen H., Martensson B. Tooth position measurements on dental casts using holographic images. Am J Orthod Dentofacial Orthop.1982;81:310-313.

3. Takade K., Lowe AA., DeCou R. Operational performance of the Reflex Metrograph and its applicability to the three-dimensional analysis of dental casts. Am J Orthod Dentofacial Orthop.1983;83:195-199.

4. SohmuraT., Kojima T., Wakabayashi K., and Takahashi J. Use of an ultrahigh-speed laser scanner for constructing three-dimensional shapes of dentition and occlusion. J. Prosthet. Dent. 2002;84:345-352.

5. Santoro M., Galkin S., Teredesai M., Nicolay OF. and Cangialosi TJ. Comparison of measurements made on digital and plaster models. Am J Orthod Dentofacial Orthop 2003;124:101-105.

6. Whetten JL, Williamson PC, Heo G, Varnhagen C and Major PW. Variations in orthodontic treatment planning decisions of Class II patients between virtual 3-dimensional models and traditional plaster study models. Am J Orthod Dentofacial Orthop. 2006;130:485-491.

7. El-Zanaty HM, El-Beialy AR, Abou El-Ezz AM, Attia KH, El-Bialy AR, Mostafa YA. Three-dimensional dental measurements: An alternative to plaster models. Am J Orthod Dentofacial Orthop. 2010;137:259-65.

8. Baumgaertel S, Palomo JM, Palomo L and Hans MG. Reliability and accuracy of cone-beam computed tomography dental measurements. Am J Orthod Dentofacial Orthop. 2009;136:19-25.

9. SPSS (2008): Statistical Package for the Social Sciences, Release 17.0.0, August 23, 2008.

10. Oremus M., Oremus C., Hall GBC., McKinnon MC. Inter-rater and testretest reliability of quality assessments by novice student raters using the Jadad and Newcastle-Ottawa Scales. BMJ 2012;2:1-6. 
Egyptian

Orthodontic Journal

11. Profit WR., Fields HW., Sarver DM. Contemporary orthodontics. $4^{\text {th }}$ ed. St. Louis: Mosby; 2007, chapter six page 201.

12. Brown AA., Scarfe WC., Scheetz JP., Silveria AM., Farman AG. Linear accuracy of Cone Beam CT derived 3D images. Angle Orthod. 2009;79:150-157.

13. Lascala CA., Panella J. and Marques MM. Analysis of the accuracy of linear measurements obtained by cone beam computed tomography (CBCT-NewTom). Dentomaxillofac Radiol 2004;33:291-294.

14. Naidu D., Freer TJ. Validity, reliability, and reproducibility of the iOC intraoral scanner: A comparison of tooth widths and Bolton ratios. Am J Orthod Dentofacial Orthop. 2013;144:304-310.

15. Zilberman O., Huggare JAV.and Parikakis KA. Evaluation of the validity of tooth size and arch width measurements using conventional and three-dimensional virtual orthodontic models. Angle Orthod. 2003;73:301-306.

16. Tehranchi A., Nouri M., Massudi R., Katchooi M., Rahimi H. Diagnostic value of manual and computerized methods of dental casts analysis. J of Dentistry, Tehran University of Medical Sciences. 2009;6:85- 90 .

17. Periago DR., Scarfe WC., Moshiri M., Scheetz JP., Silveria AM., Farman AG. Linear accuracy and reliability of Cone Beam CT derived 3-Dimensional images constructed using an orthodontic volumetric rendering program. Angle Orthod. 2007;78:387-395.

18. Champagne M. Reliability of measurements from photocopies of study models. J Clin Orthod 1992;26:648-650.

19. Mayers M., Firestone AR., Rashid R., Vig KWL. Comparison of peer assessment rating (PAR) index scores of plaster and computer-based digital models. Am J Orthod Dentofacial Orthop 2005;128:431-434. 